\title{
A Novel Method for Solving Nonlinear Volterra Integro-Differential Equation Systems
}

\author{
Mohammad Hossein Daliri Birjandi, Jafar Saberi-Nadjafi $\mathbb{D}^{D}$, and Asghar Ghorbani \\ Department of Applied Mathematics, School of Mathematical Sciences, Ferdowsi University of Mashhad, Mashhad, Iran \\ Correspondence should be addressed to Jafar Saberi-Nadjafi; najafil41@gmail.com
}

Received 12 December 2017; Accepted 2 April 2018; Published 3 June 2018

Academic Editor: Patricia J. Y. Wong

Copyright (c) 2018 Mohammad Hossein Daliri Birjandi et al. This is an open access article distributed under the Creative Commons Attribution License, which permits unrestricted use, distribution, and reproduction in any medium, provided the original work is properly cited.

\begin{abstract}
An efficient iteration method is introduced and used for solving a type of system of nonlinear Volterra integro-differential equations. The scheme is based on a combination of the spectral collocation technique and the parametric iteration method. This method is easy to implement and requires no tedious computational work. Some numerical examples are presented to show the validity and efficiency of the proposed method in comparison with the corresponding exact solutions.
\end{abstract}

\section{Introduction}

Systems of integro-differential equations and their solutions play a pivotal role in the fields of science, industrial mathematics, control theory of financial mathematics, and engineering [1-3]. Physical systems, such as biological applications in population dynamics, and genetics where impulses arise naturally or are caused by control are modeled by a system of integro-differential equations $[4,5]$. The initial value problem for a nonlinear system of integro-differential equations were used to model the competition between tumor cells and the immune system [6]. In [7], two systems of specific inhomogeneous integro-differential equations are studied in order to examine the noise term phenomenon. Thus applications of numerical methods for solving these equations are attractive. This has led to a great deal of research in recent years with the use of numerical methods such as the variational iteration method [8], differential transform method [9], Bezier curves method [10], radial basis function networks [11], biorthogonal systems [12], the block pulse functions method [13], and a collocation method in combination with operational matrices of Bernstein polynomials [14].

The parametric iteration method (PIM) is an analytic approximate method that provides the solution of linear and nonlinear problems as a sequence of iterations. In fact, the PIM as a fixed- point iteration method is a reconstruction of the variational iteration method. Since the implementation of the PIM generally leads to the calculation of unneeded terms, where more time is consumed in repeated calculations for series solutions, so to overcome these shortcomings, a useful improvement of the PIM was proposed in [15].

The aim of this work is to present an effective algorithm, requiring no tedious computational work, based on the improved PIM and the spectral collocation technique to obtaining an accurate solution for the following system of Volterra integro-differential equations as follows:

$$
\begin{aligned}
\dot{y}_{i}(t)= & f_{i}\left(t, y_{1}(t), y_{2}(t), \ldots, y_{n}(t)\right) \\
& +\int_{0}^{t} k_{i}(t, s) F_{i}\left(y_{1}(s), y_{2}(s), \ldots, y_{n}(s)\right) d s, \\
y_{i}(0)= & \left(y_{0}\right)_{i},
\end{aligned}
$$

for $i=1, \ldots, n$ and $t \in[0, T]$. The functions $f_{i}\left(t, y_{1}(t)\right.$, $\left.y_{2}(t), \ldots, y_{n}(t)\right)$ are given real valued functions, $k_{i}(t, s), i=1$, $2, \ldots, n$, are kernels of the integral equations, and $F_{i}\left(y_{1}(s)\right.$, $\left.y_{2}(s), \ldots, y_{n}(s)\right)$ are linear or nonlinear functions of $y_{1}(s)$, $y_{2}(s), \ldots, y_{n}(s)$.

To demonstrate the utility of the proposed method, some examples of system of Volterra integro-differential equations are given, which are solved using the established method. The 
obtained results are compared with the exact solutions. In all cases, the present algorithm performed excellently.

\section{Parametric Iteration Method}

The PIM gives rapidly convergence by using successive approximations of the exact solution if such a solution exists; otherwise the approximations can be used for numerical aims. To convey the basic idea of this method, we first consider (1) as below:

$$
L\left[y_{i}(t)\right]+N\left[y_{i}(t)\right]=g_{i}(t), \quad i=1, \ldots, n,
$$

where $L$ denotes the auxiliary linear operator with respect to $y_{i}$. In (2) $N$ is a nonlinear continuous operator with respect to $y_{i}$ and $g_{i}(t)$ is the source term.

Next, we construct a family of explicit iterative processes for $(2)[15,16]$

$$
L\left[y_{i}^{k+1}(t)-y_{i}^{k}(t)\right]=h A\left[y_{i}^{k}(t)\right], \quad i=1, \ldots, n,
$$

where

$$
\begin{aligned}
& A\left[y_{i}^{k}(t)\right]=L\left[y_{i}^{k}(t)\right]+N\left[y_{i}^{k}(t)\right]-g_{i}(t) \\
& \equiv \dot{y}_{i}^{k}(t)-f_{i}\left(t, y_{1}^{k}(t), y_{2}^{k}(t), \ldots, y_{n}^{k}(t)\right) \\
& \quad-\int_{0}^{t} k_{i}(t, s) F_{i}\left(y_{1}^{k}(s), y_{2}^{k}(s), \ldots, y_{n}^{k}(s)\right) d s,
\end{aligned}
$$

with the initial conditions $y_{i}^{k+1}(0)=y_{i}^{0}$.

Also, we can construct a family of the implicit PIM for (2) as follows:

$$
\begin{aligned}
L & {\left[y_{i}^{k+1}(t)-y_{i}^{k}(t)\right] } \\
& =h\left\{L\left[y_{i}^{k}(t)\right]+N\left[y_{i}^{k+1}\right]-g_{i}(t)\right\}, \quad i=1, \ldots, n,
\end{aligned}
$$

with the above initial condition.

$y_{i}^{0}$ is the initial guess which can be freely found from solving its corresponding linear equation $\left(L\left[y_{i}^{0}\right]=0\right.$ or $\left.L\left[y_{i}^{0}\right]=g_{i}(t)\right)$ and the subscript $k$ denotes the $k$ th iteration. The parameter $h \neq 0$ denotes the so-called auxiliary parameter, which can be identified easily and efficiently by the technique proposed in [15]. Also we are free to choose the auxiliary linear operator $L$, the auxiliary parameter $h$, and the initial approximation $y_{i}^{0}$, which is fundamental to the validity and flexibility of the PIM. Accordingly, the successive approximations of $y_{i}^{k}(t), k \geq 0$, for the PIM iterative relation will be obtained readily in the auxiliary parameter $h$. Consequently, the exact solution can be obtained by using the following:

$$
y_{i}(t)=\lim _{k \rightarrow \infty} y_{i}^{k}(t), \quad i=1, \ldots, n .
$$

When the original PIM fails, then the presence of the parameter $h$ in (3) or (5) can play an important role in the frame of the PIM. However, we can always discover a valid region of $h$ for every physical problem by plotting the solution or its derivatives versus the parameter $h$ in some points. An approximate optimal value of the convergence accelerating parameter $h$ can be determined at the order of approximation by the residual error [17]:

$$
\begin{aligned}
& \operatorname{Res}_{i}(h) \\
& \quad=\int_{0}^{T}\left\{L\left[y_{i}^{k}(t ; h)\right]+N\left[y_{i}^{k}(t ; h)\right]-g_{i}(t)\right\}^{2} d t .
\end{aligned}
$$

Now, one can minimize (7) by imposing the requirement $d \operatorname{Res}_{i}(h) / d h=0$.

\section{Description of the Method}

The PIM procedure provides the solution of the system of Volterra integro-differential equations as a sequence of iterates; its successive iterations may be very complex so that the resulting integrals in its iterative relation may not be performed analytically. Here, we will overcome this shortcoming of the PIM for solving (1) by proposing a spectral collocation PIM. As will be shown in this paper later, the new method will be very simple to implement and save time and calculations.

Assume that $y_{i}(t), i=1, \ldots, n$, are the $i$ th component of the solution $\mathbf{y}$ and also the $i$ th equation of system (1). Consider basis functions $\phi_{i, j}$, which are polynomials of degree $N-1$ satisfying $\phi_{i, j}\left(t_{k}\right)=\delta_{j, k}^{i}$ for the shifted Chebyshev nodes (note that $t_{1}=T$ and $t_{N}=0$ ):

$$
\begin{aligned}
& t_{k}=\frac{T}{2}\left[\cos \left(\frac{(k-1) \pi}{N-1}\right)+1\right], \\
& k=1, \ldots, N, i=1, \ldots, n .
\end{aligned}
$$

The unknown function $y_{i}(t)(i=1, \ldots, n)$ is approximated as a truncated series of polynomials. The polynomial

$$
p_{i}(t) \cong y_{i}(t)=\sum_{j=1}^{N} \varphi_{i, j}(t) y_{i, j}
$$

interpolates the points $\left(t_{j}, y_{i, j}\right), j=1, \ldots, N$, that is, $p_{i}(\mathbf{t})=$ $\mathbf{y}_{i}$, where $\mathbf{t}=\left(t_{1}, \ldots, t_{N}\right)$ and $\mathbf{y}_{i}=\left(y_{1}, \ldots, y_{N}\right)$ are vectors. The values of the interpolating polynomial of first derivative at the nodes are $\dot{p}_{i}(\mathbf{t})=D^{(1)} \mathbf{y}_{i}, i=1, \ldots, n$, where the $k$,jth element of the differentiation matrix $D^{(1)}$ is $\phi_{i, j}^{(1)}\left(t_{k}\right)$. Also the values of the integrals at the nodes are defined by $\int_{0}^{t} k_{i}(t, s) y(s) d s=V \cdot \mathbf{y}_{i}, i=1, \ldots, n$, where $V$ is the Volterra integration matrix $[18,19]$ (for more details observe [19]).

Generally, in order to solve system (1) using a spectral collocation scheme, the interpolating polynomials $p_{i}(t)(i=$ $1, \ldots, n)$ are required to satisfy the equations of the system at the interior nodes. The values of the interpolating polynomials at the interior nodes $t_{2}, \ldots, t_{N}$ are $p_{i}\left(\mathbf{t}_{m}\right)=\left(\mathbf{y}_{i}\right)_{m}=$ $I_{m,:} \mathbf{y}_{i}(m=1: N-1)$, where $I_{m,:}$ denotes the $m$ row of the $N \times N$ identity matrix and the derivative values are $\dot{p}_{i}\left(\mathbf{t}_{m}\right)=$ $D_{m, \mathrm{y}}^{(1)} \mathbf{y}_{i}$. The initial condition that involves the interpolating polynomials can be handled by using the formulas $p_{i}\left(\mathbf{t}_{N}\right)=$ 
TABLE 1: The $L_{2}$ and $L_{\infty}$ errors for Example 1 .

\begin{tabular}{lcccccc}
\hline$N$ & 6 & 8 & 10 & 12 & 14 \\
\hline$L_{2}-$ error $y_{1}(t)$ & $3.1 e-005$ & $1.4 e-007$ & $4.3 e-10$ & $9.0 e-13$ & $8.5 e-16$ & $2.0 e-15$ \\
$L_{\infty}-$ error $y_{1}(t)$ & $2.0 e-005$ & $8.1 e-008$ & $2.2 e-10$ & $4.1 e-13$ & $4.4 e-16$ & $8.8 e-16$ \\
$L_{2}-$ error $y_{2}(t)$ & $8.4 e-006$ & $2.3 e-008$ & $6.7 e-11$ & $1.3 e-13$ & $9.6 e-16$ & $1.1 e-15$ \\
$L_{\infty}-$ error $y_{2}(t)$ & $6.7 e-006$ & $1.5 e-008$ & $4.4 e-11$ & $8.4 e-14$ & $4.4 e-16$ & $6.6 e-16$ \\
\hline No. of Itre. & 23 & 21 & 31 & 17 & 21 \\
\hline
\end{tabular}

$\left(\mathbf{y}_{i}\right)_{N}=I_{N,:} \mathbf{y}_{i}$, where this notation $I_{N, \text { : }}$ denotes the last row of the $(N \times N)$ identity matrix.

For the interpolating polynomial to satisfy the $i$ th differential equation of the system of Volterra integro-differential equations (1) at each interior node, the collocation equation

$$
\begin{aligned}
\dot{p}_{i}\left(\mathbf{t}_{m}\right)= & f_{i}\left(\mathbf{t}_{m}, p_{1}\left(\mathbf{t}_{m}\right), \ldots, p_{n}\left(\mathbf{t}_{m}\right)\right) \\
& +\int_{o}^{t} k_{i}(\mathbf{t}, s) F_{i}\left(p_{1}(s), \ldots, p_{n}(s)\right) d s, \\
p_{i}\left(\mathbf{t}_{N}\right)= & y_{i}^{0}, \quad i=1, \ldots, n
\end{aligned}
$$

should be satisfied. Substituting the differentiation and the integration matrix relations into (10), we get

$$
\begin{aligned}
{\left[\begin{array}{c}
D_{m,:}^{(1)} \\
I_{N,:}
\end{array}\right] \mathbf{y}_{i}=} & {\left[\begin{array}{c}
I_{m,:} f_{i}\left(\mathbf{t}_{m}, \mathbf{y}_{1}, \ldots, \mathbf{y}_{n}\right) \\
y_{i}^{0}
\end{array}\right] } \\
& +\left[\begin{array}{c}
I_{m,:}\left(V \cdot F_{i}\left(\mathbf{y}_{1}, \ldots, \mathbf{y}_{n}\right)\right) \\
0
\end{array}\right], \\
& i=1, \ldots, n .
\end{aligned}
$$

Now, in view of (3) and the definitions of $L$ and $A$, by substituting the differentiation and integration matrix relations, we will have the following explicit PIM for solving (1) which is called the spectral PIM (SPIM):

$$
\begin{aligned}
\mathbf{y}_{i}^{k+1} & =\mathbf{y}_{i}^{k}+h\left[\begin{array}{c}
D_{m,:}^{(1)} \\
I_{N,:}
\end{array}\right]^{-1}\left(\left[\begin{array}{c}
D_{m,:}^{(1)} \\
I_{N,:}
\end{array}\right] \mathbf{y}_{i}^{k}\right. \\
- & {\left[\begin{array}{c}
I_{m,:} f_{i}\left(\mathbf{t}_{m}, \mathbf{y}_{1}, \ldots, \mathbf{y}_{n}\right) \\
y_{i}^{0}
\end{array}\right] } \\
- & {\left.\left[\begin{array}{c}
I_{m,:}\left(V \cdot F_{i}\left(\mathbf{y}_{1}, \ldots, \mathbf{y}_{n}\right)\right) \\
0
\end{array}\right]\right) . }
\end{aligned}
$$

If we define $\mathbf{L}=\left[D_{m ;:}^{(1)}, I_{N,:}\right]^{T}, \mathbf{f}=\left[f_{i}\left(\mathbf{t}_{m}, \mathbf{y}_{1}, \ldots, \mathbf{y}_{n}\right), y_{i}^{0}\right]^{T}$, and $\mathbf{N y}_{k}=\left[I_{m,:}\left(V \cdot F_{i}\left(\mathbf{y}_{1}, \ldots, \mathbf{y}_{n}\right)\right), 0\right]^{T}$, then we will have the following explicit iterative relation for finding the solution vector $\mathbf{y}_{i}^{k+1}$

$$
\mathbf{y}_{i}^{k+1}=\mathbf{y}_{i}^{k}+h \mathbf{L}^{-1}\left(\mathbf{L} \mathbf{y}_{i}^{k}-\mathbf{f}-\mathbf{N} \mathbf{y}_{i}^{k}\right) .
$$

Here the vector $\mathbf{y}_{i}^{k+1}$ is defined as

$$
\mathbf{y}_{i}^{k+1}=\left\{y_{i}^{k+1}\left(t_{1}\right), \ldots, y_{i}^{k+1}\left(t_{N}\right)\right\} .
$$

Now one can start with the initial guess $\mathbf{y}_{i}^{0}$ for obtaining the approximations $\mathbf{y}_{i}$.

\section{Illustrative Examples}

In this section, we give several test examples to confirm our analysis. To examine the accuracy of the results, $L_{2}$ and $L_{\infty}$ are employed to assess the efficiency of the method SPIM. All the computations were performed using software Matlab and terminated when the current iterate satisfies $\| \mathbf{y}_{k}-$ $\mathbf{y}_{k-1} \| \leq 10^{-16}$, where $\mathbf{y}_{k}$ is the solution vector of the $k$ th SPIM iteration.

Example 1. Consider the following system of Volterra integro-differential equations [20]:

$$
\begin{gathered}
\dot{y}_{1}(t)=1-\frac{1}{2} \dot{y}_{2}^{2}(t) \\
+\int_{0}^{t}\left((t-s) y_{2}(s)+y_{2}(s) y_{1}(s)\right) d s, \\
\dot{y}_{2}(t)=2 t+\int_{0}^{t}\left((t-s) y_{1}(s)-y_{2}^{2}(s)+y_{1}^{2}(s)\right) d s,
\end{gathered}
$$

with the initial conditions $y_{1}(0)=0$ and $y_{2}(0)=1$. The exact solution of this system is $\left(y_{1}(t), y_{2}(t)\right)=(\sinh t, \cosh t)$.

Table 1 illustrates the $L_{2}$ and $L_{\infty}$ errors for different values of $N$ as well as the number of iterations to reach the abovementioned stopping criteria. Also Figure 1 shows the absolute error of the proposed method for $N=16$. As expected, the exponential rate of convergence is observed for the system of nonlinear Volterra integro-differential equations, which confirmed our theoretical predictions.

Example 2. Consider the following system of Volterra integro-differential equations with the exact solution $\left(y_{1}(t), y_{2}(t)\right)=\left(t+e^{t}, t-e^{t}\right)[8]$ :

$$
\begin{aligned}
& \dot{y}_{1}(t)=1+t-t^{2}-y_{2}(t)+\int_{0}^{t}\left(y_{1}(s)+y_{2}(s)\right) d s, \\
& \dot{y}_{2}(t)=-1-t+y_{1}(t)-\int_{0}^{t}\left(y_{1}(s)-y_{2}(s)\right) d s,
\end{aligned}
$$

subjected to initial conditions $y_{1}(0)=1$ and $y_{2}(0)=-1$.

The results of $L_{2}$ and $L_{\infty}$ errors of $y_{1}(t)$ and $y_{2}(t)$ for the different values of $N$ as well as the number of iterations to reach the stopping criteria are given in Table 2. Figure 2 depicts the absolute error of the presented method for $N=16$. Again, the exponential rate of convergence is observed for the system of nonlinear Volterra integrodifferential equations. 

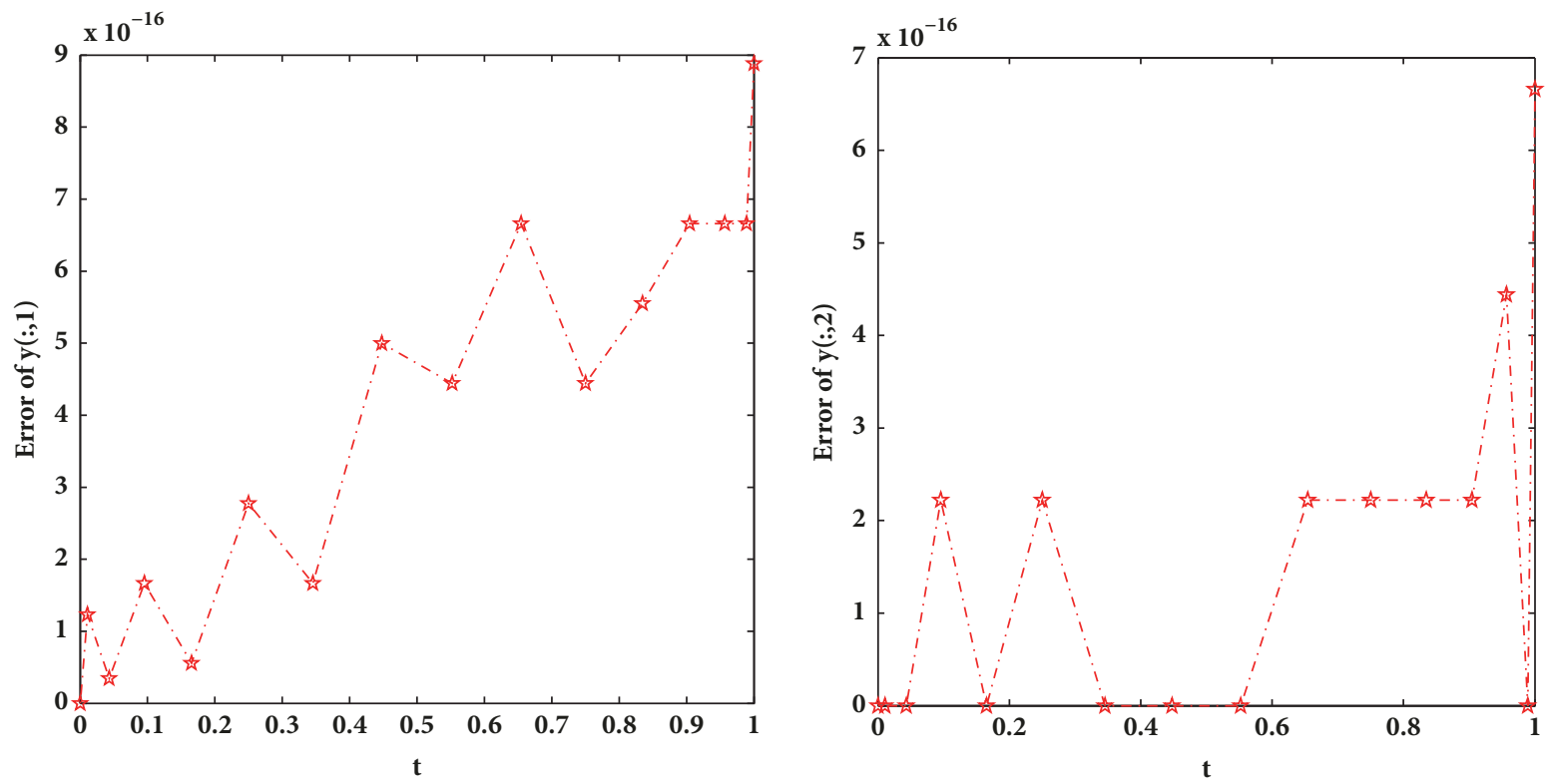

FIgURE 1: Errors of $y_{1}$ and $y_{2}$ for Example 1 when $N=16$.
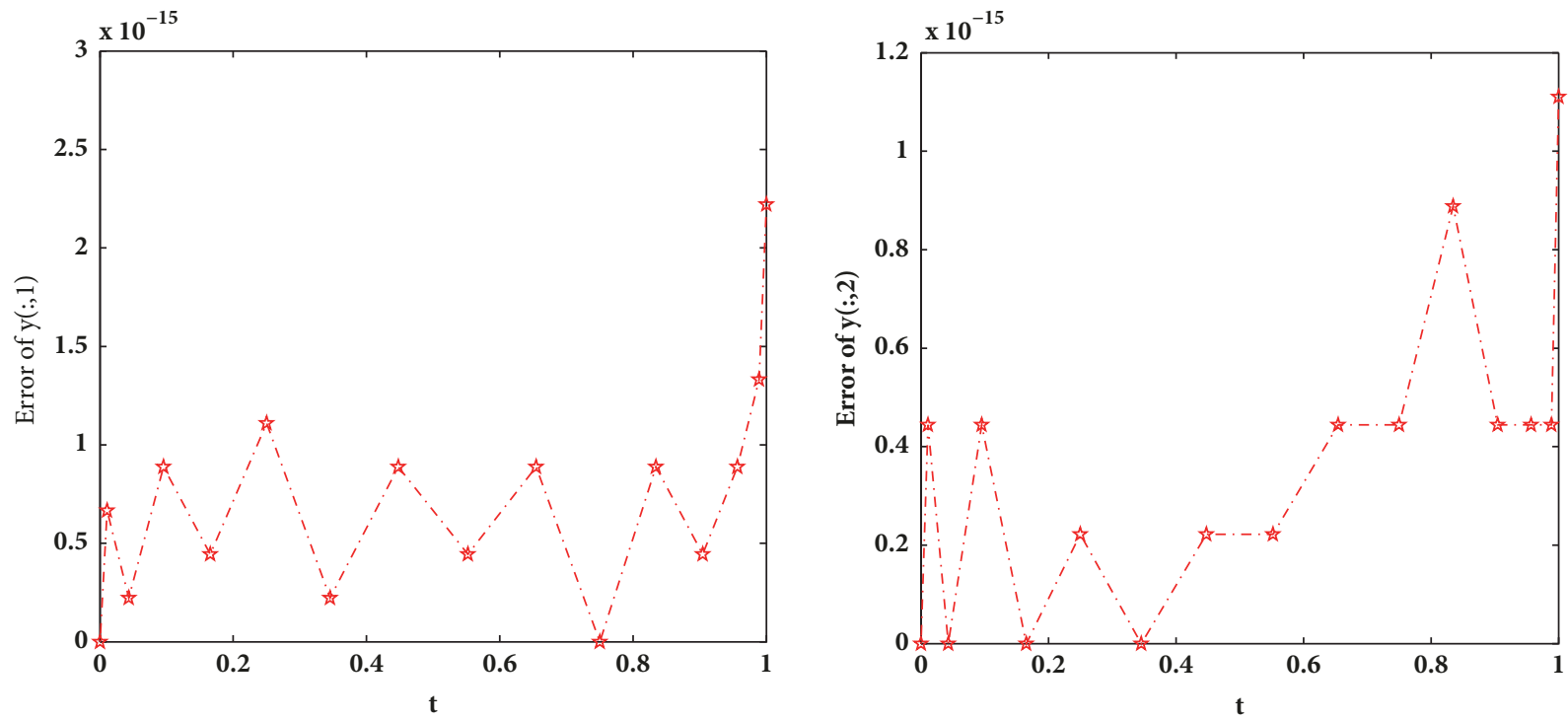

Figure 2: Errors of $y_{1}$ and $y_{2}$ for Example 2 when $N=16$.

TABLE 2: The $L_{2}$ and $L_{\infty}$ errors for Example 2.

\begin{tabular}{lcccccc}
\hline$N$ & 6 & 8 & 10 & 12 & 14 \\
\hline$L_{2}-$ errory $_{1}(t)$ & $2.0 e-005$ & $2.5 e-008$ & $1.8 e-11$ & $1.0 e-14$ & $4.3 e-15$ & $5.5 e-15$ \\
$L_{\infty}-$ errory $_{1}(t)$ & $1.2 e-005$ & $1.2 e-008$ & $8.2 e-12$ & $3.9 e-15$ & $2.6 e-15$ & $2.6 e-15$ \\
$L_{2}-$ errory $_{2}(t)$ & $1.3 e-005$ & $1.6 e-008$ & $1.3 e-11$ & $6.8 e-15$ & $1.4 e-15$ & $2.1 e-15$ \\
$L_{\infty}-$ errory $_{2}(t)$ & $8.9 e-006$ & $1.0 e-008$ & $7.1 e-12$ & $3.1 e-15$ & $8.8 e-16$ & $1.1 e-15$ \\
\hline No. of Itre. & 28 & 22 & 22 & 29 & 24 \\
\hline
\end{tabular}


TABLE 3: The $L_{2}$ and $L_{\infty}$ errors for Example 3 when $N=16$.

\begin{tabular}{lcccccc}
\hline$N$ & 6 & 8 & 10 & 12 & 14 \\
\hline$L_{2}-$ errory $_{1}(t)$ & $1.9 e-004$ & $9.7 e-007$ & $6.4 e-008$ & $4.8 e-10$ & $8.0 e-12$ & $6.9 e-14$ \\
$L_{\infty}-$ errory $_{1}(t)$ & $1.2 e-004$ & $5.5 e-007$ & $3.1 e-008$ & $2.2 e-10$ & $3.2 e-12$ & $2.7 e-14$ \\
$L_{2}-$ errory $_{2}(t)$ & $1.8 e-005$ & $4.2 e-007$ & $8.2 e-009$ & $5.9 e-11$ & $1.3 e-12$ & $2.5 e-15$ \\
$L_{\infty}-$ errory $_{2}(t)$ & $1.2 e-005$ & $2.9 e-007$ & $5.3 e-009$ & $3.3 e-11$ & $7.6 e-13$ & $1.1 e-15$ \\
\hline No. of Itre. & 23 & 18 & 17 & 16 & 15 \\
\hline
\end{tabular}
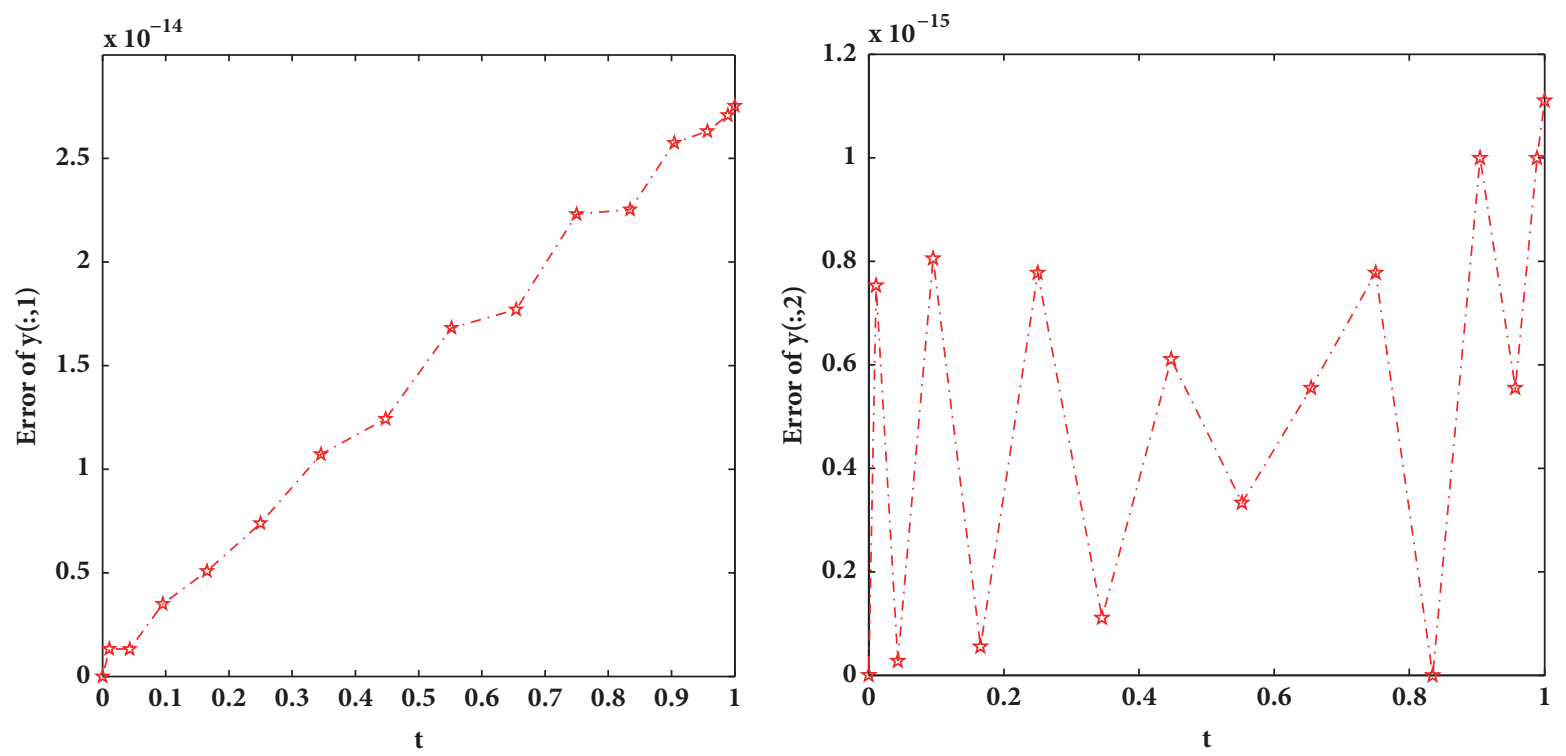

FIgURE 3: Errors of $y_{1}$ and $y_{2}$ for Example 3 when $N=16$.

Example 3. Finally, let us consider the following system of nonlinear Volterra integro-differential equations [20]:

$$
\begin{aligned}
\dot{y}_{1}(t)= & 2 y_{2}(t)-\frac{1}{3} t^{4}+\cos \left(y_{1}(t)\right)-1 \\
& +\int_{0}^{t}\left(2 s \sin \left(y_{1}(s)\right)+s t y_{2}(s)\right) d s, \\
\dot{y}_{2}(t)= & 1-t \sin \left(y_{2}(t)\right)-\frac{1}{2} t^{2} \sin \left(y_{1}(t)\right) \\
& +\int_{0}^{t}\left(s t^{2} \cos \left(y_{1}(s)\right)+t \cos \left(y_{2}(s)\right)\right) d s,
\end{aligned}
$$

with the initial conditions of $y_{1}(0)=0$ and $y_{2}(0)=0$ whose exact solution is given by $y_{1}(t)=t^{2}$ and $y_{2}(t)=t$.

The errors and number of iterations for the different values of $N$ are presented in Table 3. The other results are presented in Figure 3 with $N=16$ similar to Examples 1 and 2.

\section{Conclusion}

In this paper, we presented a powerful numerical approach based on a combination of the Chebyshev spectral collocation technique and the parametric iteration method for solving the linear and nonlinear system of Volterra integrodifferential equations. This method inherits the strengths of the PIM and is easy to implement and is accurate when applied to the linear and nonlinear system of Volterra integrodifferential equations. The comparison of the approximate solution and the exact solution reveals that the present method is very accurate and convenient for solving the linear and nonlinear system of Volterra integro-differential equations.

\section{Conflicts of Interest}

Mohammad Hossein Daliri Birjandi, Jafar Saberi-Nadjafi, and Asghar Ghorbani declare that there are no conflicts of interest regarding the publication of this paper.

\section{References}

[1] M. A. Jaswon and G. T. Symm, Integral Equation Methods in Potential Theory and Elastostatics, Academic Press, London, UK, 1977.

[2] S. Hatamzadeh-Varmazyar, M. Naser-Moghadasi, E. Babolian, and Z. Masouri, "Numerical approach to survey the problem of electromagnetic scattering from resistive strips based on using a set of orthogonal basis functions," Progress in Electromagnetics Research, vol. 81, pp. 393-412, 2008. 
[3] A. Kyselka, "Properties of systems of integro-differential equations in the statistics of polymer chains," Polymer Science U.S.S.R., vol. 19, no. 11, pp. 2852-2858, 1977.

[4] S. Kheybari, M. T. Darvishi, and A. M. Wazwaz, "A semianalytical algorithm to solve systems of integro-differential equations under mixed boundary conditions," Journal of Computational and Applied Mathematics, vol. 317, pp. 72-89, 2017.

[5] S. Kheybari, M. T. Darvishi, and A. M. Wazwaz, "A semianalytical approach to solve integro-differential equations," Journal of Computational and Applied Mathematics, vol. 317, pp. 17-30, 2017.

[6] N. Bellomo, B. Firmani, and L. Guerri, "Bifurcation analysis for a nonlinear system of integro-differential equations modelling tumor-immune cells competition," Applied Mathematics Letters, vol. 12, no. 2, pp. 39-44, 1999.

[7] A.-M. Wazwaz, "The existence of noise terms for systems of inhomogeneous differential and integral equations," Applied Mathematics and Computation, vol. 146, no. 1, pp. 81-92, 2003.

[8] J. Saberi-Nadjafi and M. Tamamgar, "The variational iteration method: a highly promising method for solving the system of integro-differential equations," Computers \& Mathematics with Applications, vol. 56, no. 2, pp. 346-351, 2008.

[9] A. Arikoglu and I. Ozkol, "Solutions of integral and integro-differential equation systems by using differential transform method," Computers \& Mathematics with Applications, vol. 56, no. 9, pp. 2411-2417, 2008.

[10] F. Ghomanjani, A. Kiliçman, and S. Effati, "Numerical solution for IVP in volterra type linear integrodifferential equations system," Abstract and Applied Analysis, vol. 2013, Article ID 490689, 2013.

[11] A. Golbabai, M. Mammadov, and S. Seifollahi, "Solving a system of nonlinear integral equations by an RBF network," Computers \& Mathematics with Applications. An International Journal, vol. 57, no. 10, pp. 1651-1658, 2009.

[12] M. I. Berenguer, A. I. Garralda-Guillem, and M. Ruiz Guiz Galán, "An approximation method for solving systems of Volterra integro-differential equations," Applied Numerical Mathematics, vol. 67, pp. 126-135, 2013.

[13] K. Maleknejad, H. Safdari, and M. Nouri, "Numerical solution of an integral equations system of the first kind by using an operational matrix with block pulse functions," International Journal of Systems Science, vol. 42, no. 1, pp. 195-199, 2011.

[14] K. Maleknejad, B. Basirat, and E. Hashemizadeh, "A Bernstein operational matrix approach for solving a system of high order linear Volterra-Fredholm integro-differential equations," Mathematical and Computer Modelling, vol. 55, no. 3-4, pp. 1363-1372, 2012.

[15] A. Ghorbani and J. Saberi-Nadjafi, "A piecewise-spectral parametric iteration method for solving the nonlinear chaotic Genesio system," Mathematical and Computer Modelling, vol. 54, no. 1-2, pp. 131-139, 2011.

[16] S. A. Alavi, A. Heydari, and F. Khellat, "On convergence and error analysis of the parametric iteration method," International Journal of Applied Mathematical Research, vol. 4, no. 1, p. 129, 2014.

[17] A. Ghorbani and M. Gachpazan, "A Spectral Quasilinearization Parametric Method for Nonlinear Two-Point Boundary Value Problems," Bulletin of the Malaysian Mathematical Sciences Society.

[18] J. A. Weideman and S. C. Reddy, "A MATLAB differentiation matrix suite," ACM Transactions on Mathematical Software, vol. 26, no. 4, pp. 465-519, 2000.
[19] T. Damian, Matrix Based Operatorial Approach to Differential and Integral Problems, Babes Bolyai University of Cluj-Napoca, Romania, 2011.

[20] M. I. Berenguer, A. I. Garralda-Guillem et al., "An approximation method for solving systems of Volterra integro-differential equations," Applied Numerical Mathematics, vol. 67, pp. 126-135, 2013. 


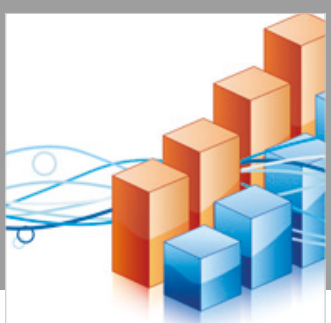

Advances in

Operations Research

\section{-n-m}
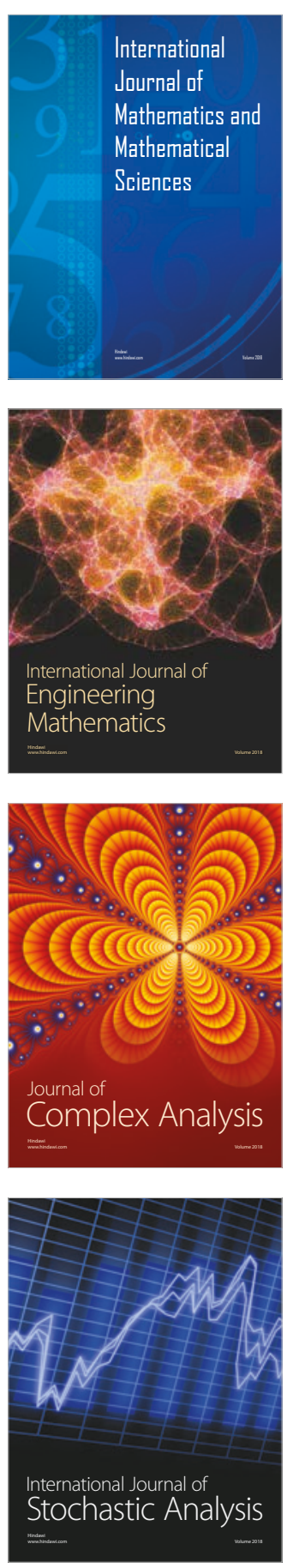
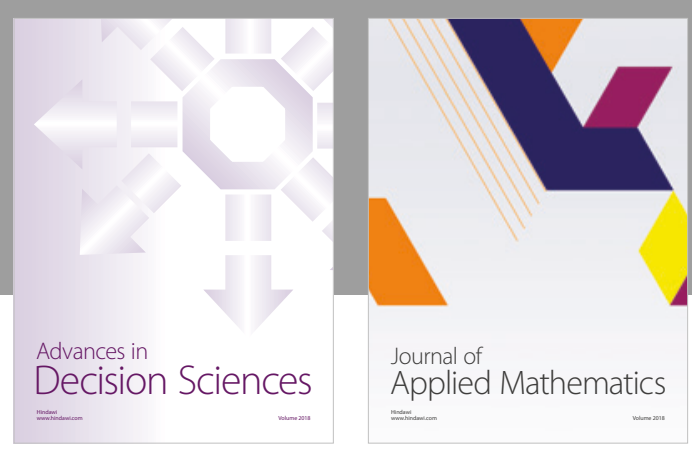

Journal of

Applied Mathematics
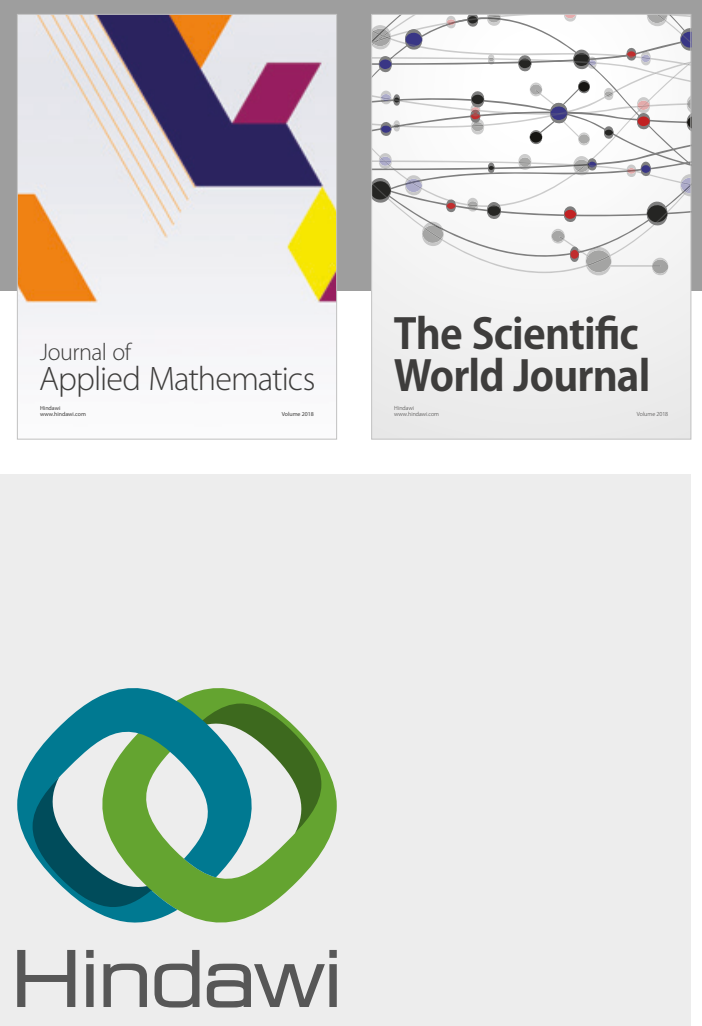

Submit your manuscripts at

www.hindawi.com

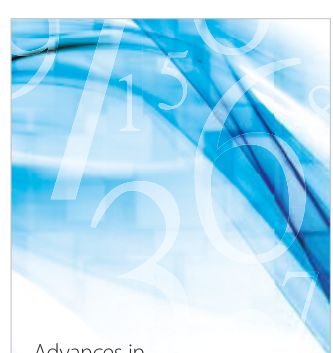

Advances in
Numerical Analysis
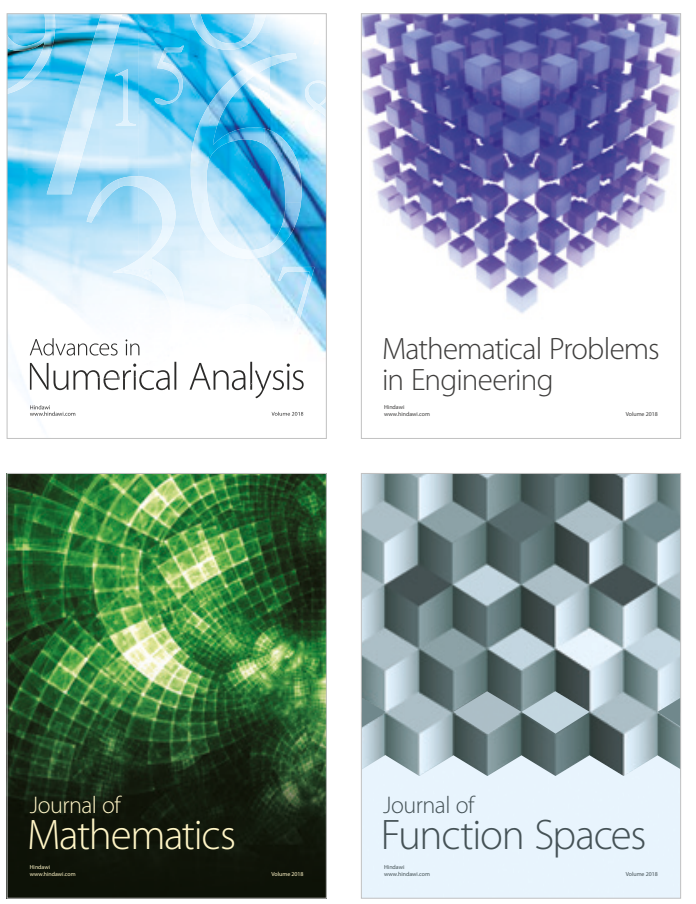

Mathematical Problems in Engineering

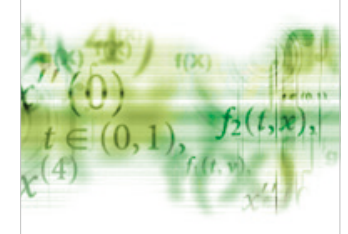

International Journal of

Differential Equations

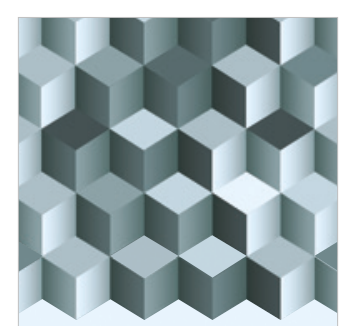

Journal of

Function Spaces

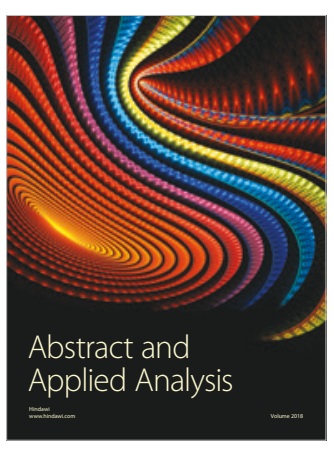

The Scientific

World Journal

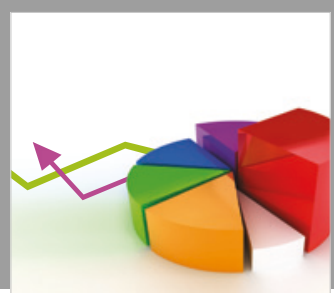

Journal of

Probability and Statistics
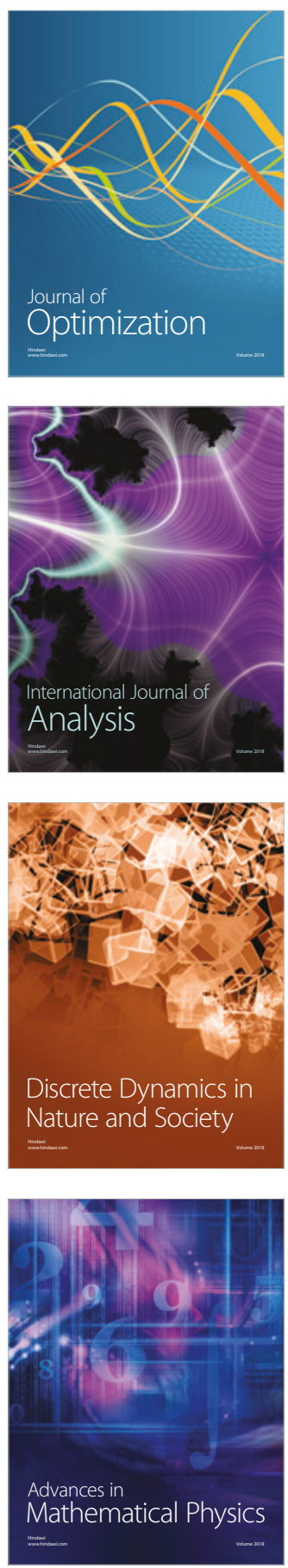